\title{
THE THOM SPACE PERIODICITY OF CLASSIFYING SPACES ${ }^{1}$
}

\author{
DENIS SJERVE
}

\begin{abstract}
If $G$ is any topological group then there exists a classifying space $B_{G}$. In this paper we shall exhibit a fiber bundle $\omega$ over $B_{G}$ such that the Thom complex $B_{G}^{\omega}$ is homeomorphic to $B_{G}$. As an application we give a new proof of the Freudenthal Suspension Theorem.
\end{abstract}

1. Introduction. As a starting point for this paper consider the sequence of complex projective spaces $C P^{k}$ together with their canonical complex line bundles $\omega_{k}$. These bundles are compatible with respect to the usual inclusions $C P^{m} \mapsto C P^{k}$, and moreover there exist homeomorphisms $\left(C P^{k}\right)^{\omega_{k}} \cong C P^{k+1}$ also compatible with inclusions [1] (here $X^{\omega}$ denotes the Thom complex of $\omega$ over $X)$. Taking the limit as $k \rightarrow \infty$ we obtain a homeomorphism $\left(C P^{\infty}\right)^{\omega} \cong$ $C P^{\infty}$, where $\omega$ is the universal complex line bundle. This homeomorphism reveals a geometrically periodic structure on $C P^{\infty}$ since the Thom complex $\left(C P^{\infty}\right)^{\omega}$ is like $C P^{\infty}$ with a shift in dimensions.

For another example of periodicity consider the sequence of quaternionic projective spaces $H P^{k}$ together with the associated quaternionic line bundles $\omega_{k}$. Then again there exist homeomorphisms $\left(H P^{k}\right)^{\omega_{k}} \cong H P^{k+1}$ compatible with inclusions $H P^{m} \mapsto H P^{k}$, and they produce in the limit a homeomorphism $\left(H P^{\infty}\right)^{\omega} \cong H P^{\infty}$, where $\omega$ is now the universal quaternionic line bundle [1]. Therefore $H P^{\infty}$ also has a geometrically periodic structure.

These two examples tend to suggest that any classifying space $B_{G}$, where $G$ is any topological group, should carry some kind of periodic structure. In fact, in $\S 2$ we shall construct a fiber bundle pair $\omega$ :

$$
(C G, G) \mapsto(D(\omega), S(\omega)) \rightarrow B_{G},
$$

where $C$ denotes the unreduced cone functor, with the property that the quotient complex $D(\omega) / S(\omega)$ is naturally homeomorphic to $B_{G}$. To be explicit, the bundle $\omega$ is the $(C G, G)$ bundle associated to Milnor's universal principal $G$ bundle $E_{G} \rightarrow B_{G}$ [3] by the obvious $G$ action on $(C G, G)$. Then $D(\omega)-S(\omega)$ is the $C G-G$ bundle associated to the action of $G$ on the open cone $C G-G$. Thus we consider the pair $(D(\omega), S(\omega))$ as a generalization of

Received by the editors December 1, 1975 and, in revised form, May 17, 1976.

AMS (MOS) subject classifications (1970). Primary 55F35, 55H10; Secondary 55D40.

Key words and phrases. Classifying spaces, Thom complexes, Milnor join construction, periodicity.

${ }^{1}$ This paper was given as a lecture at the second annual Ohio State University Topology Conference, August 25-29, 1975. The author would like to express his gratitude to Ohio State University and in particular to Professors Henry Glover and Guido Mislin. 
the disc bundle-sphere bundle pair of a vector bundle. Accordingly we shall define the Thom complex $B_{G}^{\omega}$ to be $D(\omega) / S(\omega)$.

In $\$ 3$ we shall exploit the relative Serre spectral sequence of the bundle pair $\omega$ to give a type of algebraic periodicity for $H_{*}\left(B_{G}\right)$ and $H^{*}\left(B_{G}\right)$. One particular consequence of this spectral sequence will be a new proof of the Freudenthal Suspension Theorem.

2. Universal bundles and periodicity. Throughout this section $G$ shall denote a completely arbitrary topological group. In order to construct the bundle $\omega$ over $B_{G}$ we recall the join construction of Milnor [3]. Thus we let $G$ act on the $k$-fold joins $G^{k}=G * \cdots * G$ by

$$
G^{k} \times G \rightarrow G^{k}, \quad\left(t_{1} g_{1}+\cdots+t_{k} g_{k}, g\right) \rightarrow t_{1} g_{1} g+\cdots+t_{k} g_{k} g .
$$

Then by taking the quotient spaces $X_{k}=G^{k} / G$ we obtain for each $k \geqslant 1$ a principal $G$ bundle $p_{k}: G^{k} \rightarrow X_{k}$. These bundles are obviously compatible with the inclusions $G^{m} \rightarrow G^{k}$ and so yield in the limit a universal principal $G$ bundle $p_{\infty}: G^{\infty} \rightarrow X_{\infty}$. Following standard terminology we shall write $E_{G}$ for $G^{\infty}$ and $B_{G}$ for $X_{\infty}$. Thus we have a commutative diagram of principal bundles

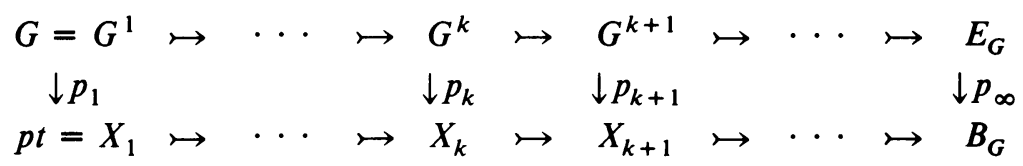

To any of the bundles $p_{k}: G^{k} \rightarrow \dot{X}_{k}, 1 \leqslant k \leqslant \infty$, we now associate the fiber bundle pair $\omega_{k}$ whose total space is given by

$$
\left(D\left(\omega_{k}\right), S\left(\omega_{k}\right)\right)=G_{G}^{k} \times(C G, G)
$$

and whose projection $\left(D\left(\omega_{k}\right), S\left(\omega_{k}\right)\right) \rightarrow X_{k}$ is induced by $p_{k}: G^{k} \rightarrow X_{k}$. Then we clearly have $\omega_{k} \mid X_{m}=\omega_{m}$ for $m \leqslant k$ and so we shall usually drop the subscript from the notation. In particular we have constructed the fiber bundle pair $\omega$ :

$$
\begin{aligned}
(C G, G) \stackrel{i}{\rightarrow} & (D(\omega), S(\omega)) \rightarrow B_{G}, \\
& \text { where }(D(\omega), S(\omega))=\left(E_{G} \times(C G, G)\right) / G .
\end{aligned}
$$

Notice that $\left(D\left(\omega_{1}\right), S\left(\omega_{1}\right)\right)=G \times{ }_{G}(C G, G)$ may be naturally identified with $(C G, G)$ by the map

$$
G \underset{G}{\times} C G \rightarrow C G, \quad\left[g,\left[t, g^{\prime}\right]\right] \rightarrow\left[t, g^{\prime} g^{-1}\right] .
$$

We adopt the notation that square brackets shall always denote equivalence classes. Since $X_{1}$ is a natural base point for $B_{G}$ we take the inclusion $\left(D\left(\omega_{1}\right)\right.$, $\left.S\left(\omega_{1}\right)\right) \mapsto(D(\omega), S(\omega))$ to be the fiber map $(C G, G) \mapsto^{i}(D(\omega), S(\omega))$ of $(2.2)$.

(2.3) The Geometric Periodicity Theorem. For each $k, 1 \leqslant k \leqslant \infty$, the Thom complex $X_{k}^{\omega}$ is naturally homeomorphic to $X_{k+1}$. In particular there exists a homeomorphism $B_{G}^{\omega} \cong B_{G}$ which is functorial in $G$. 
Proof. First consider the case $k<\infty$. A typical point of $D\left(\omega_{k}\right)=G^{k}$ $\times_{G} C G$ is $\left[t_{1} g_{1}+\cdots+t_{k} g_{k},[t, g]\right]$ and so we can define a map $\Phi^{\prime}$ : $D\left(\omega_{k}\right) \rightarrow X_{k+1}$ by

$$
\Phi^{\prime}\left[t_{1} g_{1}+\cdots+t_{k} g_{k},[t, g]\right]=\left[(1-t) g+t t_{1} g_{1}+\cdots+t t_{k} g_{k}\right] .
$$

The subspace of $D\left(\omega_{k}\right)$ given by setting $t=0$ is mapped to a point. But this subspace is just $S\left(\omega_{k}\right)$ and so $\Phi^{\prime}$ induces a map $\Phi: D\left(\omega_{k}\right) / S\left(\omega_{k}\right) \rightarrow X_{k+1}$.

To construct an inverse of $\Phi$ consider the set $A$ of all points $\left[u_{1} h_{1}\right.$ $\left.+\cdots+u_{k+1} h_{k+1}\right] \in X_{k+1}$ such that $0 \leqslant u_{1}<1$. In other words $A=X_{k+1}$ $-X_{1}=X_{k+1}-p t$. Also $A$ is the image set $\Phi^{\prime}\left(D\left(\omega_{k}\right)-S\left(\omega_{k}\right)\right)$ and we can define a map $\Psi^{\prime}: A \rightarrow D\left(\omega_{k}\right)-S\left(\omega_{k}\right)$ by

$$
\begin{array}{r}
\Psi^{\prime}\left[u_{1} h_{1}+\cdots+u_{k+1} h_{k+1}\right]=\left[t_{1} g_{1}+\cdots+t_{k} g_{k},[t, g]\right] \\
\text { where } t_{i} g_{i}=\left(u_{i+1} /\left(1-u_{1}\right)\right) h_{i+1}, t=1-u_{1}, g=h_{1} .
\end{array}
$$

Then $\Psi^{\prime}$ extends to a map $\Psi: X_{k+1} \rightarrow D\left(\omega_{k}\right) / S\left(\omega_{k}\right)$ and one can easily check that $\Psi$ is the inverse of $\Phi$. The homeomorphisms $X_{k}^{\omega_{k}} \cong X_{k+1}$ so constructed are compatible with respect to the inclusions $X_{k} \mapsto X_{m}$, and so produce in the limit a homeomorphism $X_{\infty}^{\omega} \cong X_{\infty}$. This proves (2.3).

The fiber map $i:(C G, G) \mapsto(D(\omega), S(\omega))$ and the homeomorphism $\Phi$ : $D(\omega) / S(\omega) \cong B_{G}$ determine a map $\phi: \Sigma G \rightarrow B_{G}$, where $\Sigma$ is the unreduced suspension functor.

(2.4) LEMMA. $\phi$ is the standard inclusion of $X_{2}$ into $B_{G}$.

By applying the relative Serre spectral sequence to the fiber bundle pair (2.2) and using the homeomorphism $D(\omega) / S(\omega) \cong B_{G}$ of (2.3) we can derive

(2.5) The Algebraic Periodicity Theorem. Suppose $G$ is a connected topological group. Then there exist spectral sequences which are functorial in $G$.

$$
\begin{aligned}
& E_{s, t}^{2} \cong H_{s}\left(B_{G} ; \tilde{H}_{t}(\Sigma G)\right) \Rightarrow \tilde{H}_{s+t}\left(B_{G}\right), \\
& E_{2}^{s, t} \cong H^{s}\left(B_{G} ; \tilde{H}^{t}(\Sigma G)\right) \Rightarrow \tilde{H}^{s+t}\left(B_{G}\right) .
\end{aligned}
$$

If $G$ is either $S^{1}$ or $S^{3}$ then $\omega$ is either the universal complex line bundle or the universal quaternionic line bundle and (2.3) reduces to the examples at the beginning of this paper. Moreover the spectral sequences of (2.5) collapse totally giving isomorphisms

$$
\begin{array}{r}
H_{s}\left(B_{G}\right) \cong \tilde{H}_{s+n}\left(B_{G}\right), \quad H^{s}\left(B_{G}\right) \cong \tilde{H}^{s+n}\left(B_{G}\right) \text { for all } s, \\
\text { where } n=2\left(\text { resp. 4) if } G=S^{1}\left(\text { resp. } S^{3}\right) .\right.
\end{array}
$$

The periodicity theorems can be generalized by starting with an arbitrary principal $G$ bundle $p: W \rightarrow X$ rather than with the special bundle $G \rightarrow p t$. The group $G$ acts on the $k$-fold joins $W^{k}$ producing principal $G$ bundles $p_{k}$ : $W^{k} \rightarrow X_{k}$ for all $k \geqslant 1$. Taking the limit we have a universal principal $G$ bundle $p_{\infty}: W^{\infty} \rightarrow X_{\infty}$ and a commutative diagram of principal $G$ bundles 


$$
\begin{aligned}
& \begin{array}{l}
W=W^{1} \mapsto \cdots \\
\downarrow p_{1}
\end{array} W^{k} \mapsto W^{k+1} \mapsto \cdots \mapsto W^{\infty} \\
& X=X_{1} \mapsto \cdots \mapsto X_{k} \mapsto X_{k+1} \mapsto \cdots \mapsto X_{\infty}
\end{aligned}
$$

Then for $1 \leqslant k \leqslant \infty$ we can construct a bundle pair $\omega_{k}$ over $X_{k}$,

$$
(C W, W) \mapsto\left(D\left(\omega_{k}\right), S\left(\omega_{k}\right)\right) \rightarrow X_{k}
$$

by defining $\left(D\left(\omega_{k}\right), S\left(\omega_{k}\right)\right)=W^{k} \times{ }_{G}(C W, W)$ and taking for the projection the map induced by $p_{k}: W^{k} \rightarrow X_{k}$. The proof of (2.3) now yields a homeomorphism $X_{k}^{\omega_{k}} \cong X_{k+1} / X_{1}$ compatible with inclusions. Thus we have proved

(2.7) TheOREM. Suppose $p: W \rightarrow X$ is a principal $G$ bundle. Then there exists a fiber bundle pair $\omega$ :

$$
(C W, W) \mapsto(D(\omega), S(\omega)) \rightarrow X_{\infty}
$$

and a periodicity homeomorphism $X_{\infty}^{\omega} \cong X_{\infty} / X_{1}$. Moreover the construction of $\omega$ and the periodicity are functorial for maps between principal bundles.

Algebraic periodicity then follows from the relative Serre spectral sequence.

(2.8) THEOREM. Suppose $p: W \rightarrow X$ is a principal $G$ bundle with $W$ connected. Then there are spectral sequences

$$
\begin{aligned}
& E_{s, t}^{2} \cong H_{s}\left(X_{\infty} ; H_{t}(C W, W)\right) \Rightarrow H_{s+t}\left(X_{\infty}, X_{1}\right), \\
& E_{2}^{s, t} \cong H^{s}\left(X_{\infty} ; H^{t}(C W, W)\right) \Rightarrow H^{s+t}\left(X_{\infty}, X_{1}\right),
\end{aligned}
$$

which are functorial for maps between principal bundles.

In [4], the periodicity theorems, (2.7) and (2.8), were proved for the case where $p: W \rightarrow X$ is a regular covering space with deck transformation group $G$. The essential idea of the proof in that paper was to define and construct normal bundles $\omega_{k}$ for the embeddings $X_{k} \mapsto X_{k+1}$. That approach carries over to this paper to give another proof of (2.7).

3. Applications. As an application of the homology spectral sequence in (2.5) we shall prove the Freudenthal Suspension Theorem. To do this we need the simplicial loop space construction of Milnor [2]. If $G(X)$ is the space of simplicial loops and $E(X)$ is the space of simplicial paths then $G(X)$ is a topological group and there exists a principal universal $G(X)$ bundle $E(X) \rightarrow$ $X$. This bundle is equivalent to the Serre path-loop fibration $P(X) \rightarrow X$ since the obvious inclusion $E(X) \mapsto P(X)$ is compatible with respect to projections onto $X$ and produces a homotopy equivalence $G(X) \mapsto \Omega(X)$.

Taking $G$ to be $G(X)$ in (2.2) and (2.3) we see that we have a fiber bundle pair $\omega$ :

$$
(C G(X), G(X)) \stackrel{i}{\mapsto}(D(\omega), S(\omega)) \rightarrow B_{G(X)}
$$

such that $D(\omega) / S(\omega) \cong B_{G(X)}$. Since $G(X) \simeq \Omega(X)$ and $B_{G(X)} \simeq X$ the homology spectral sequence of $(2.5)$ becomes 


$$
E_{s, t}^{2} \cong H_{s}\left(X ; \tilde{H}_{t}(\Sigma \Omega X)\right) \Rightarrow \tilde{H}_{s+t}(X) .
$$

Before studying the structure of this spectral sequence we must determine the fiber map $\Sigma \Omega(X) \rightarrow X$. By (2.4) the fiber map $i:(C G(X), G(X)) \rightarrow(D(\omega)$, $S(\omega))$ determines the map $\phi: \Sigma G(X) \rightarrow B_{G(X)}, \phi[t, g]=[(1-t) g+t e]$. Then composing with the particular homotopy equivalence $B_{G(X)} \simeq X$ constructed in [5] proves

(3.3) Lemma. The fiber map of the spectral sequence (3.2) is the map $v$ : $\Sigma \Omega(X) \rightarrow X$ defined by $v[t, \omega]=\omega(1-t)$.

(3.4) TheOREM. Suppose $X$ is a $k$ connected countable simplicial complex, where $k>1$. Then $v_{*}: H_{n}(\Sigma \Omega X) \rightarrow H_{n}(X)$ is an isomorphism for $n \leqslant 2 k$ and an epimorphism for $n \leqslant 2 k+1$.

Proof. In terms of the spectral sequence (3.2), $v_{*}$ is the composite

$$
\tilde{H}_{n}(\Sigma \Omega X) \cong E_{0, n}^{2} \rightarrow E_{0, n}^{\infty} \mapsto \tilde{H}_{n}(X) \text {. }
$$

For any $r, 2 \leqslant r \leqslant \infty$, we have $E_{s, t}^{r}=0$ if either $0<s \leqslant k$ or $t \leqslant k$. In particular, we always have

$$
E_{1, n-1}^{\infty}=\cdots=E_{k, n-k}^{\infty}=0 .
$$

On the other hand we will also have

$$
E_{k+1, n-k-1}^{\infty}=E_{k+2, n-k-2}^{\infty}=\cdots=0
$$

if $n-k-1 \leqslant k$. Thus we have proved that if $n \leqslant 2 k+1$ then $E_{0, n}^{\infty} \cong$ $H_{n}(X)$ and so $v_{*}$ is an epimorphism for this range of dimensions.

To prove that $E_{0, n}^{2}=E_{0, n}^{\infty}$ consider the differentials at $E_{0, n}^{r}$ :

$$
E_{r, n-r+1}^{r} \stackrel{d^{r}}{\rightarrow} E_{0, n}^{r} \stackrel{d^{r}}{\rightarrow} E_{-r, n+r-1}^{r}=0 .
$$

If $n-r+1 \leqslant k$ for all $r \geqslant k+1$ then we have $E_{0, n}^{2}=E_{0, n}^{\infty}$. Thus we have proved that if $n \leqslant 2 k$ then $v_{*}$ is an isomorphism. This concludes the proof.

To relate (3.4) to the Freudenthal Suspension Theorem define $\mu=\mu_{Y}$ : $Y \rightarrow \Omega \Sigma Y$ to be the map given by $\mu(y)(t)=[t, y]$. Also define $\lambda=\lambda_{Y}$ : $\Sigma \Omega Y \rightarrow Y$ by $\lambda[t, \omega]=\omega(t)$. Then, by a standard fact about adjoint functors, the composite $\lambda_{\Sigma Y} \circ \Sigma \mu_{Y}: \Sigma Y \rightarrow \Sigma Y$ is the identity.

(3.5) Corollary. Suppose $X$ is a $k$ connected countable simplicial complex, where $k \geqslant 0$. Then $\mu_{*}: H_{n}(X) \rightarrow H_{n}(\Omega \Sigma X)$ is an isomorphism for all $n \leqslant 2 k$ +1 .

Proof. $\lambda$ and $v$ are maps $\Sigma \Omega X \rightarrow X$ that differ by a self-homeomorphism of $\Sigma \Omega X$ and therefore they have the same connectivity properties. In particular, (3.4) implies that $\left(\lambda_{\Sigma X}\right)_{*}: H_{n}(\Sigma \Omega \Sigma X) \rightarrow H_{n}(\Sigma X)$ is an isomorphism for $n \leqslant 2 k+2$ and an epimorphism for $n \leqslant 2 k+3$. It follows that $\left(\Sigma \mu_{X}\right)_{*}$ : $H_{n}(\Sigma X) \rightarrow H_{n}(\Sigma \Omega \Sigma X)$ is an isomorphism for $n \leqslant 2 k+2$, and this proves (3.5).

In the usual manner one can now derive the Freudenthal Suspension Theorem. 
(3.6) COROLlaRY. Suppose $X$ is an $k$ connected countable simplicial complex, where $k>1$. Then the suspension homomorphism $\Sigma: \pi_{n}(X) \rightarrow \pi_{n+1}(\Sigma X)$ is an isomorphism for $n<2 k$ and an epimorphism for $n<2 k+1$.

\section{REFERENCES}

1. M. F. Atiyah, Thom complexes, Proc. London Math. Soc. (3) 11 (1961), 291-310. MR 24 \#A1727.

2. J. Milnor, Construction of universal bundles. I, Ann. of Math. (2) 63 (1956), 272-284. MR 17, 994.

3. __ Construction of universal bundles. II, Ann. of Math. (2) 63 (1956), 430-436. MR 17, 1120.

4. D. Sjerve, Group actions and generalized periodicity, Math. Z. 149 (1976), 1-11.

5. J. Stasheff, Homotopy associativity of $H$ spaces. I, II, Trans. Amer. Math. Soc. 108 (1963), 293-312. MR 28 \# 1623.

Department of Mathematics, University of Brttish Columbia, Vancouver, British Columbia, Canada 